\title{
Formation of Twins During Thermal Fatigue of Magnesium Wrought Alloy AZ31*
}

\author{
Martin KRAUSS** and Berthold SCHOLTES** \\ ${ }^{* *}$ Department of Mechanical Engineering, Institute of Materials Engineering, \\ University of Kassel, Mönchebergstr. 3, 34109 Kassel \\ Germany, E-mail: Scholtes@uni-kassel.de
}

\begin{abstract}
Results of thermal fatigue tests of the magnesium base alloy AZ31 for the case of out-of-phase-loading in a temperature range between $-50{ }^{\circ} \mathrm{C}$ and $+290^{\circ} \mathrm{C}$ are presented. Specimens were loaded under constant total strain and uniaxial homogeneous stress. The thermal fatigue behavior is described by the resulting stress amplitudes, plastic strain amplitudes and mean stresses as a function of the number of thermal loading cycles. It is well known that rolled AZ31 shows different stress-strain behavior during tensile and compressive loading at lower temperatures due to the fact that mechanical twinning occurs preferentially during the compression loading phase and not during tension. However untwinning processes may occur during the tensile phase of a loading cycle. As a consequence, during the first thermal loading cycles, typical consequences of the formation and the dissolution of twins can be observed. The interaction of deformation, recovery and recrystallization processes, characteristic for individual temperature ranges are discussed in detail to analyze the damage progress during thermal fatigue.
\end{abstract}

Key words: Thermal Fatigue, Magnesium, Twinning, AZ31, Cyclic Deformation

\section{Introduction}

Mg-alloys are very promising materials for light-weight constructions. Unfortunately, because of the hexagonal lattice structure, their deformability in metal forming processes at room temperature is rather poor and up to now, applications of $\mathrm{Mg}$ wrought alloys have been limited. At lower temperatures, slip is only possible in parallel basal planes and twinning is an important mechanism of plastic deformation. In this case, however, for geometrical reasons, a special shear direction is necessary. In polycrystalline materials with a preferred orientation of the crystallites, as it is the case in rolled sheets, for that reason also macroscopic plastic deformation of specimens or components depends on their loading direction relative to the orientation of the parent sheet material. As a consequence, tension or compression loading yields different stress-strain curves and also different deformation induced microstructures [1-3].

In addition to mechanical loading, in practice many components are subjected to alternating temperatures. As a consequence, cyclic thermal stresses may be induced and thermal fatigue processes with characteristic failure mechanisms occur [4], depending on the specific loading conditions. In order to realistically predict strength and lifetime of such components and for safe constructions, characteristic aspects of the time-dependent damage processes as well as of microstructural alterations have to be known.

Consequently, a detailed study was conducted to analyse the behavior of the wrought Mg-alloy AZ31 and the cast Mg-alloy AZ91 during out-of-phase thermal fatigue loading conditions [5]. Only results for AZ31 are reported here. 


\section{Material investigated and experimental details}

The material investigated was the wrought Mg-alloy AZ31 (3.4 wt.-\% Al, 0.76 wt.-\% $\mathrm{Zn}$, balance $\mathrm{Mg}$ ), which was delivered as a warm rolled $20 \mathrm{~mm}$ thick sheet. Metallographic investigations of the microstructure revealed nearly completely recrystallized grains with a mean grain size of $14 \mu \mathrm{m}$. They did not show any special morphological orientation, however texture pole figures revealed a typical rolling texture of the base material with a pronounced orientation of the basal planes of the hexagonal unit cells parallel to the sheet surface. Cylindrical specimens with a diameter of $7 \mathrm{~mm}$ and a gauge length of $10 \mathrm{~mm}$ were taken in rolling direction from the central part of the sheet. Surfaces in the gauge length were fine turned and no further heat treatment was applied.

Thermo-mechanical tests were carried out using a servohydraulic computer controlled testing device. Specimens were cyclically heated by an induction coil and cooled by a mixture of compressed air and liquid nitrogen, depending on the temperature range applied. Thus a nearly triangular time-temperature course with $\mathrm{dT} / \mathrm{dt} \approx 5^{\circ} \mathrm{C} / \mathrm{s}$ was achieved.

Experiments with a lower temperature level of $-50^{\circ} \mathrm{C}$ and maximum temperatures up to $+290^{\circ} \mathrm{C}$ were carried out under total strain control (out-of-phase thermal fatigue testing) and the resulting temperature-stress-hystereses were measured. Using correct thermal expansion coefficients, also plastic strain amplitudes could be determined. To simulate the influence of different mean stresses, total strains were kept constant and experiments were started at different temperature levels, e.g. the lower, mean or upper temperature of the thermal cycle. Microstructures and the formation of cracks at different stages of the thermal fatigue process were analysed by light or scanning electron microscopy and by X-ray diffraction techniques.

\section{Results}

Figure 1 shows stress-strain curves of AZ 31 during isothermal loading at the temperatures indicated. Typical differences can be detected comparing the results of tensile and compression tests for temperatures lower than $+150^{\circ} \mathrm{C}$. While in tensile tests, decreasing temperatures lead to increasing flow stresses, this is not the case for compression loading, where the $0.2 \%$-proof stress remains nearly constant at approximately $-85 \mathrm{MPa}$. This is due to onset of mechanical twinning during compression at this stress level, which does not occur during tension because of the preferred orientation of the crystallites after rolling. Twinning is also indicated by serrated flow at the resp. temperatures during compression, but is not observed during loading in tension [6]. Because in compression at lower temperatures the

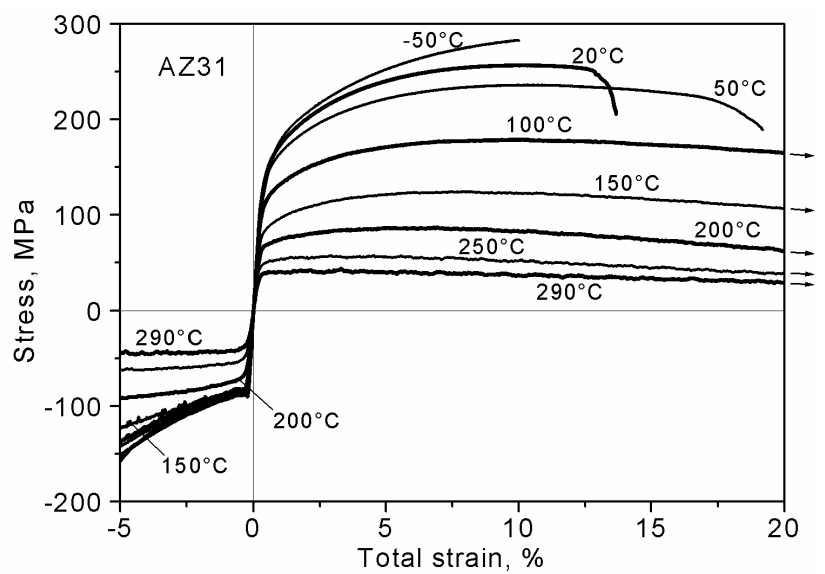

Fig. 1. Stress-strain curves of AZ31 for tensile or compression loading at the temperatures indicated. 
stress necessary for the onset of twinning is lower than the respective stress for dislocation glide in secondary slip systems, twinning is the main deformation mechanism in this case. It causes a tilt of the basal planes by nearly $90^{\circ}$ in the deformation direction and basal planes in twinned regions are oriented perpendicular to the loading axis. Microstructural investigations revealed that twinning starts in favourably oriented grains and spreads across the gauge volume similar to a Lüders band, leading to the stress plateau mentioned above. For higher plastic deformations strain hardening occurs and further grains deform by twinning and/or dislocation slip.

It has been shown that twins, which have been produced during compressive loading can vanish during a following tensile loading cycle [6]. In addition to metallographic investigations, this has been revealed by X-ray diffraction texture analyses. A texture component due to the tilting of the twinned areas could be detected after compressive loading which vanished after a subsequent tensile loading. Fig. 2 shows a stress-strain hysteresis loop at room temperature with a total strain amplitude of 3\%, starting with compression. As mentioned above, plastic deformation during compression is governed by twinning processes. After unloading and reloading of the specimen in tension, reversed twinning (untwinnig of the volume fractions twinned during compression) occurs. This is indicated by a region with a low strain hardening rate and also by serrated flow and altogether produces a characteristic shape of the hysteresis loop [6, 7].

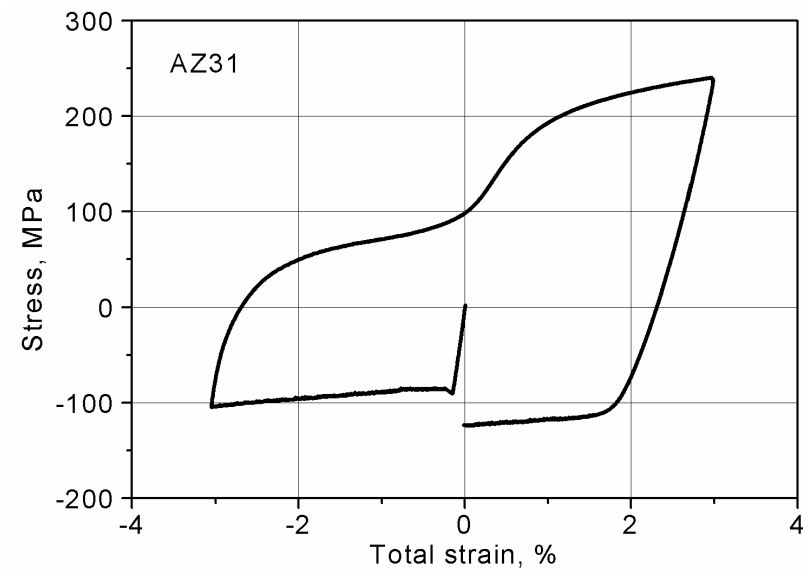

Fig. 2. Hysteresis curve of AZ31 at room temperature for the loading path indicated.

The macroscopic deformation asymmetry in uniaxial tension and compression loading described above has also characteristic consequences for the materials behavior during thermomechanical loading. This is demonstrated in Fig. 3, showing stress-temperature hysteresis loops with a temperature range $\Delta \mathrm{T}=240^{\circ} \mathrm{C}$ (temperature amplitude $120^{\circ} \mathrm{C}$ ) during the first loading cycle for three different experimental conditions, namely starting experiments at the lower, the mean or the upper temperature level of the thermal loading cycle. If experiments are started at the lower temperature of $-50^{\circ} \mathrm{C}$, at first increasing temperatures lead to increasing compressive stresses, because total strain is kept constant. At stress levels of approximately -90 MPa however, several sharp drops of the flow stress occur, which can be attributed to the localized formation of twins in favourably oriented grains. Because of the only small influence of temperature on onset of twinning in this temperature range, stress remains almost constant between $50^{\circ} \mathrm{C}$ and $100^{\circ} \mathrm{C}$. The formation of twins can also been seen in Fig. 4. It shows a micrograph of a specimen after the first thermal loading cycle. Regions with a high twin density can be detected while in other 
regions recrystallized grains without twins exist. Thus an inhomogeneous microstructure develops as a consequence of the thermal loading process.

A further example of the strongly localized formation of twins in form of twin-bands can be seen in Fig. 5. It shows the cross-section of a specimen loaded with 100 cycles between $-50^{\circ} \mathrm{C}$ and $100^{\circ} \mathrm{C}$ in an experiment started at the mean temperature. Two small twinned bands extending almost across the whole sectional area can be detected while nowhere else twins are observable. As a consequence of the localized formation of twins, also local residual stress fields develop, which have pronounced consequences on the damage process during fatigue. They lead to the formation of fatigue cracks starting at the intersection of the twinned bands with the specimen surface and, in this way, have a strong influence on fatigue life or fatigue strength.

Another important mechanism, which influences the microstructure during thermal fatigue loading of AZ31 is recrystallization. This process of course is influenced by the temperature range and the upper temperature applied, but also by the local microstructure produced during the individual loading history. Near surface areas with high dislocation

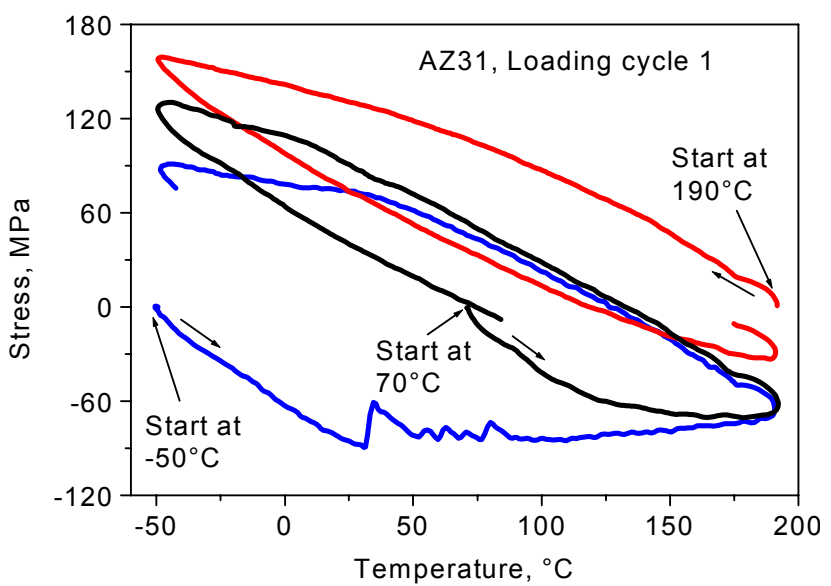

Fig. 3. Stress-temperature curves of the first loading cycle using different starting temperatures (temperature range: $240^{\circ} \mathrm{C}$ ).

densities due to the chip forming manufacturing process of the specimen start very soon to recrystallize during the thermal loading tests and develop a very fine grained approximately $15 \mu \mathrm{m}$ thick surface layer. Within the bulk of the specimens highly deformed volume fractions are also preferred regions of recrystallization processes. In this way, a very inhomogeneous microstructure with locally fluctuating mechanical properties is produced. In addition, in the same regions, periodically recrystallization or strain hardening by twinning or dislocation glide may occur during the thermal loading history.

Obviously, if thermal cycling starts at the lower temperature level, twinning is an important mechanism to control the developing microstructure and plastic deformation. After reaching the maximum temperature, during the first stage of the cooling period, nearly elastic unloading occurs (see Fig. 3). However at a temperature of approximately $40^{\circ} \mathrm{C}$, the curve starts deviating from linearity which is due to a reversed twinning (untwinning) process, leading to a lower strain hardening rate. It can be assumed that during compression loading at temperatures above $150^{\circ} \mathrm{C}$, dislocation glide considerably has contributed to plastic deformation. Hence untwinning is also influenced by the dislocation structure produced in this temperature range. As a consequence, a nearly complete reversibility of twinning, as it is observed in quasistatic compression-tension tests under isothermal conditions [6] cannot be expected because of twin-dislocation interactions. Twin-dislocation interactions may also be the reason for the lack of serrated flow during untwinning. 


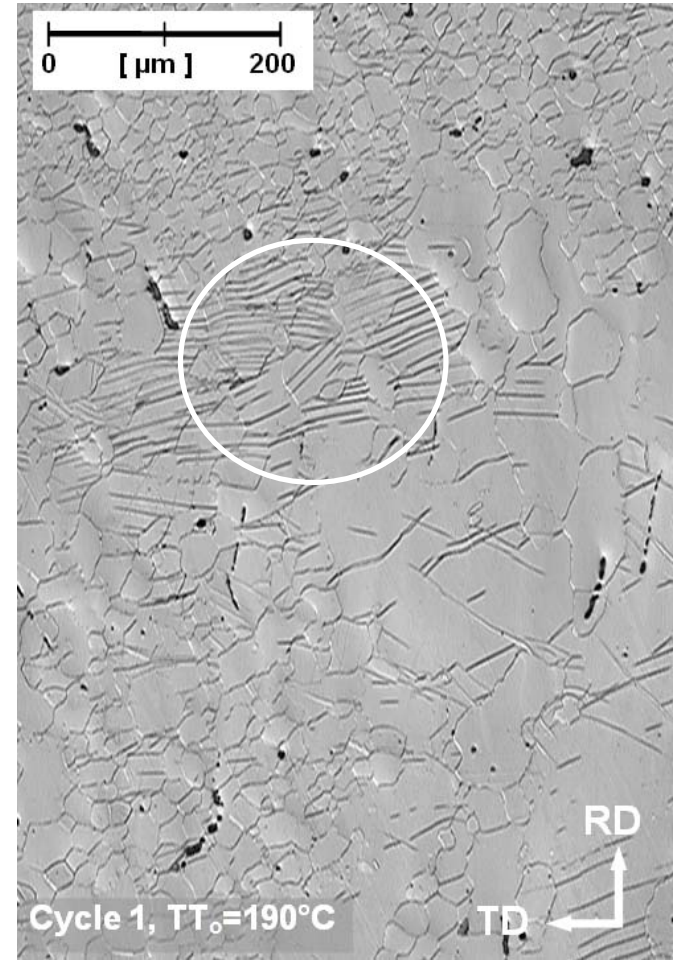

Fig. 4. Micrograph of a specimen after the first loading cycle (Temperature range $240^{\circ} \mathrm{C}$, lower temperature $-50^{\circ} \mathrm{C}$ ) showing region with high twin density.

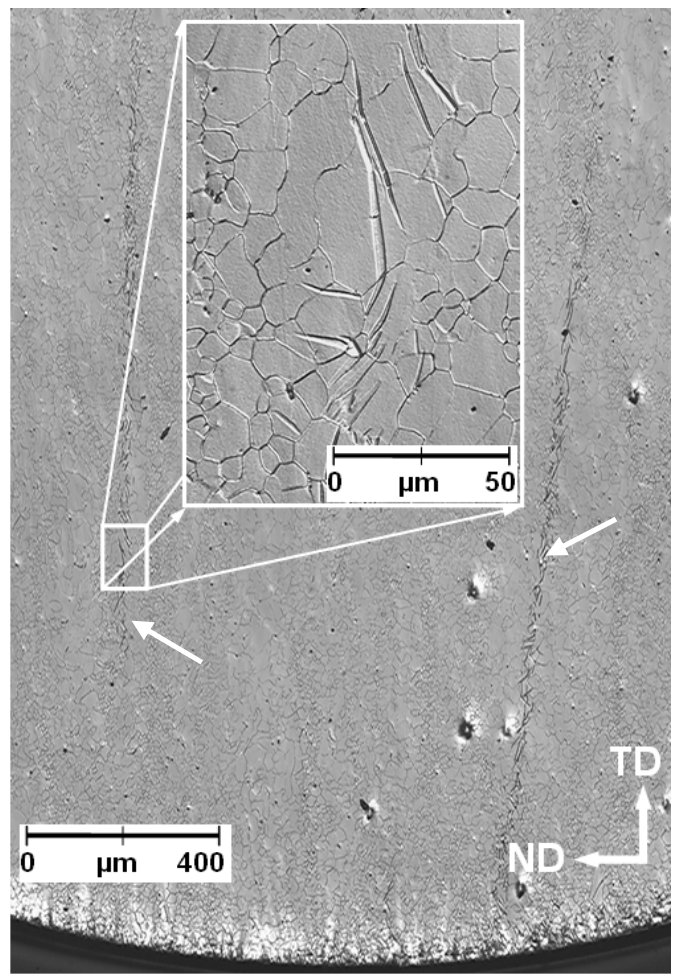

Fig. 5. Micrograph of a specimen after 100 loading cycles (Temperature range $150^{\circ} \mathrm{C}$, lower temperature $-50^{\circ} \mathrm{C}$ ) with two twinned bands. 
The untwinning process of the twinned regions, formed during the compressive part of the first loading cycle also continues during several of the following loading cycles. This is demonstrated in Fig. 6, which shows the first, second, third and $5^{\text {th }}$ loading cycle of a specimen fatigued with a temperature amplitude of $120^{\circ} \mathrm{C}$ and a starting temperature of $-50^{\circ} \mathrm{C}$. One can clearly see that, except for the $5^{\text {th }}$ loading cycle, low strain hardening regions exist in the low temperature regions, when thermally induced stresses decrease, indicating dissolution processes of formerly twinned regions. In this way untwinning reduces maximum tensile stresses during this part of the loading cycle considerably. Metallographic investigations reveal that twins did not completely disappear, but twin density is markedly reduced and lower than after the first half loading cycle.

Figure 6 also shows that soon after the first thermal loading cycle relatively stable stress-temperature hysteresis loops can be observed. Experiments with different individual starting conditions, i.e. start at the lowest, mean or highest temperature level, then only differ by the development of their mean stress levels [5].

In Fig. 7, the numbers of thermal cycles to fracture are plotted as a function of the maximum temperatures of the thermal loading cycles for experiments with a base temperature of $-50^{\circ} \mathrm{C}$. In all cases, fracture was defined by the number of thermal cycles, where the stress amplitude decreased by more than $30 \%$. With increasing maximum temperatures, lower numbers of cycles to failure or thermal fatigue lifetimes are observed. It is interesting to note that all experiments, independent of the starting condition of the experiments, can be described by the same straight line similar to a Woehler-curve. At an upper temperature of $125^{\circ} \mathrm{C}$ in some cases fracture occurred before 10.000 loading cycles were reached. For an upper temperature of $100^{\circ} \mathrm{C}$, no fracture below 10.000 cycles was observed.

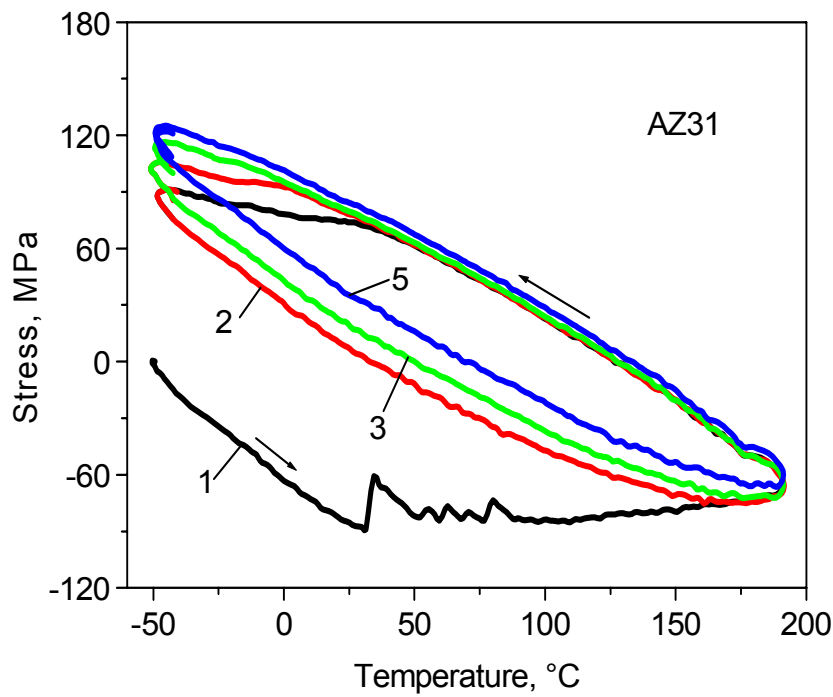

Fig 6. Stress-temperature curves of the first loading cycles for an experiment started at the lower temperature of $-50^{\circ} \mathrm{C}$ (temperature range $240^{\circ} \mathrm{C}$ ). 
In Figs. 8-10, characteristic properties of the hysteresis loops for the case "start at the lower temperature" i.e. stress amplitudes, plastic strain amplitudes and mean stresses are plotted as a function of numbers of cycles for different temperature amplitudes. In all cases, the lowest temperature of the thermal cycles is $-50^{\circ} \mathrm{C}$.

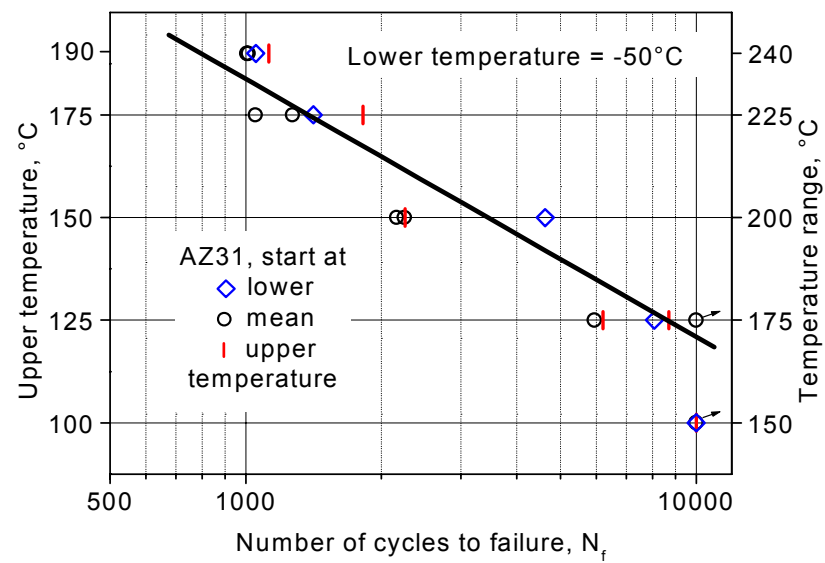

Fig. 7. Numbers of loading cycles to failure as a function of the upper temperature of the thermal cycles (lower temperature: $-50^{\circ} \mathrm{C}$ ).

In Fig. 8, stress amplitudes as a function of the numbers of thermal cycles are shown. In all cases, stresses in a range between approximately $70 \mathrm{MPa}$ and $100 \mathrm{MPa}$ were measured. Increasing upper temperatures lead to increasing stress amplitudes and, as already mentioned above, to lower lifetimes. With increasing numbers of cycles, stress amplitudes at first increase slowly, which is more pronounced for higher temperature amplitudes. Then, the stress amplitudes continuously decrease. Note that due to the logarithmic scaling, this comprises between 30 and $35 \%$ of the total lifetime of the specimens.

Plastic strain amplitudes, resulting from experiments as mentioned above, are plotted in Fig. 9 as a function of the numbers of thermal cycles. In all cases shown, high plastic strain amplitudes are observed during the first cycles of the tests. Here the influence of the forma-

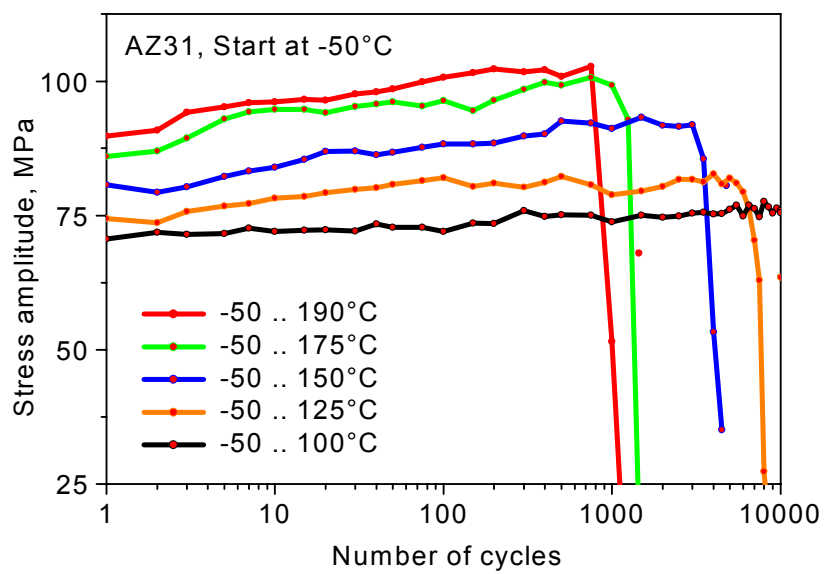

Fig. 8. Stress amplitudes as a function of the number of thermal cycles for the temperature ranges indicated (lower test temperature: $-50^{\circ} \mathrm{C}$, upper temperatures are indicated).

tion of twins can clearly be seen. Plastic strain amplitudes decrease continuously up to fracture of the specimens. They are the higher, the higher the temperature amplitude of the cyclic thermal loading process is. Obviously, the formation and disappearing of twins, 
together with recrystallization processes, leads to a stabilized microstructure with increasing numbers of loading cycles.

Figure 10 shows the development of mean stresses during the thermal cyclic loading process described. Except for the highest temperature amplitude, in all cases, at the beginning compressive mean stresses are measured. Their amounts decrease with increasing upper temperature of the thermal cycles applied. Then, tensile mean stresses built up. They rapidly increase with increasing numbers of thermal cycles and reach maximum values. Finally, during the last fraction of lifetime, they decrease until fracture occurs.

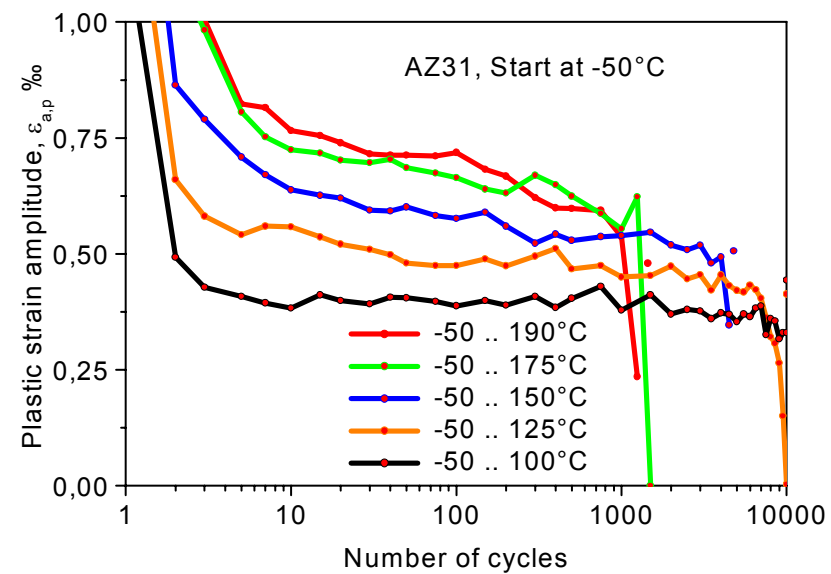

Fig. 9. Plastic strain amplitudes as a function of the number of thermal cycles for the temperature ranges indicated (lower test temperature: $-50^{\circ} \mathrm{C}$, upper temperatures are indicated).

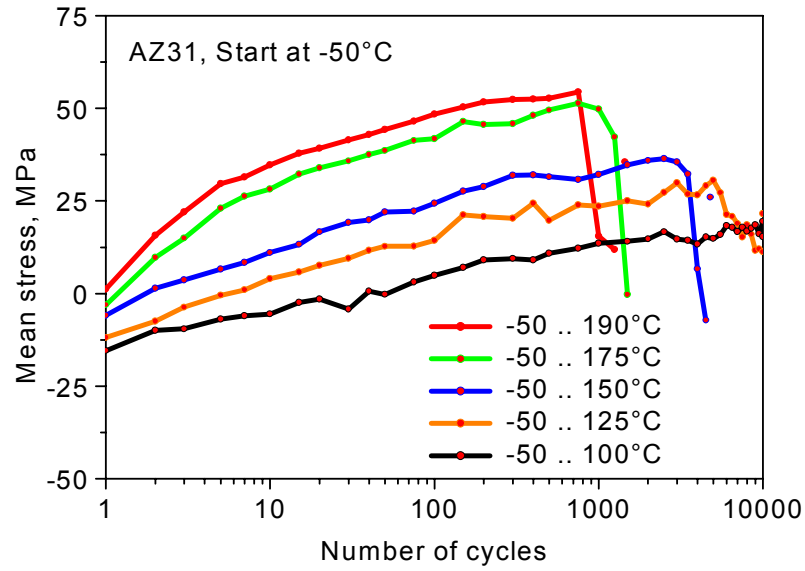

Fig. 10. Mean stresses as a function of the number of cycles for the temperature ranges indicated (lower test temperature: $-50^{\circ} \mathrm{C}$, upper temperatures are indicated).

\section{Discussion}

Depending on the temperature amplitude and the starting conditions applied, thermal fatigue is characterized by a complex history of individual stress-temperature combinations. They vary as a function of the number of cycles and determine the damage process, which finally leads to fracture. From Fig. 7, one can conclude that for experiments with the same base temperature of $-50^{\circ} \mathrm{C}$, as it is the case here, the upper temperature of the thermal loading cycle or the corresponding temperature amplitude are an unambiguous characteristic 
to describe thermal fatigue lifetime under the loading conditions applied here. The mean stresses developing during the tests are determined by the temperature amplitude as well as by the influence of the upper temperature on strength and plastic deformation of the material. Obviously, increasing upper temperatures and thus the temperature amplitude exceed the effect of temperature on strength, at least for higher numbers of cycles. Therefore mean stresses develop after a few thermal loading cycles, which are independent of the starting conditions of the experiments. The fact that during the initial stage of the experiments, the individual starting conditions have an influence on the formation of mean stresses, had no significant effect on crack initiation or propagation of cracks. This may be due to the dominant influence of fluctuating microstresses on damage development. Also it can be concluded that macrocrack propagation is only important in later stages of the fatigue life of the specimens and, hence, is not affected by mean stresses during early stage of the lifetime.

For upper temperatures between $120^{\circ} \mathrm{C}$ and $190^{\circ} \mathrm{C}$ identical dominant damage mechanisms can be assumed. Therefore, it is not surprising that the plastic strain amplitude at half the number of cycles to failure is clearly correlated with the number of cycles to failure (Coffin-Manson plot). This is shown in Fig. 11 and allows an easy assessment of fatigue lifes using measured plastic strain amplitudes.

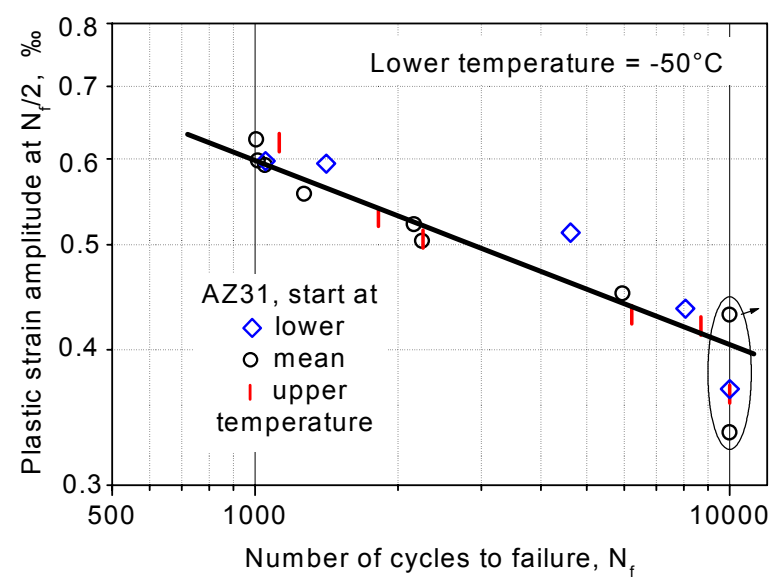

Fig. 11. Numbers of cycles to failure as a function of the plastic strain amplitude at half the number of cycles to failure for experiments with a base temperature of $-50^{\circ} \mathrm{C}$ and different temperature amplitudes.

Especially during the first loading cycles, different stress and plastic strain amplitudes result, depending on the temperature, where total strains are kept constant and experiments are started. This is clearly to be seen in Figs. 3 and 6. As a consequence, also different characteristic microstructures develop. In the case discussed here, important mechanisms, which determine the microstructure of the magnesium alloy AZ31 during thermal fatigue loading are the formation of twins, dislocation glide, recrystallization processes and their interactions. The fact that, nevertheless, thermal fatigue lifetime can be clearly correlated with upper temperatures or temperature amplitudes of the experiments can be attributed to the above mentioned interactions between the formation of deformation induced microstructures and their dissolution by recrystallization processes. Obviously, at higher numbers of cycles, this leads to microstructures which are independent of the starting conditions of the tests. Already during the first thermal loading cycle stresses observed are different from the corresponding values during isothermal tests under quasi static loading conditions and corresponding plastic deformations. The hysteresis loops shown in Fig. 3 definitely demonstrate that due to the individual temperature and deformation history, thermal fatigue processes of the material investigated here cannot be assessed using results of isothermal 
experiments alone.

The authors are grateful to the German Research Foundation DFG for financial support of the work.

\section{References}

1. U. Noster, B. Scholtes, Z. Metallkunde 94 (2003), pp. 559-563.

2. S. R. Agnew, O. Duygulu, Materials Science Forum 419-422 (2003), pp. 177-188.

3. J. Polmear, Light Alloys, Metallurgy of Light Metals, Edward Arnold, London (1981).

4. S. Suresh, Fatigue of Materials, Cambridge University Press, Cambridge (1998).

5. M. Krauß, Zur thermischen Ermüdung der Magnesiumbasislegierungen AZ31 und AZ91, Forschungsberichte aus dem Institut für Werkstofftechnik-Metallische Werkstoffe der Universität, edited by B. Scholtes, Kassel, kassel university press, Vol. 8 (2007) (in German).

6. M. Götting, B. Scholtes, Int. J. Mat. Res. 97 (2006), pp. 1378-1383.

7. L. Jiang, J. J. Jonas, Scripta Materialia 58 (2008), pp. 803-806. 